\title{
In Situ Observations of Magnetization Process in Alnico Magnets by Electron Holography and Lorentz Microscopy
}

\author{
Joong Jung Kim ${ }^{1}$, Hyun Soon Park ${ }^{1}$, Daisuke Shindo ${ }^{1, *}$, \\ Satoshi Hirosawa ${ }^{2}$ and Hideyuki Morimoto ${ }^{2}$ \\ ${ }^{1}$ Institute of Multidisciplinary Research for Advanced Materials, Tohoku University, Sendai 980-8577, Japan \\ ${ }^{2}$ NEOMAX Co., Shimamoto-cho, Mishimagun, Osaka 618-0013, Japan
}

\begin{abstract}
The magnetic microstructure of Alnico 5 and Alnico 8 and their magnetization process were investigated systematically at a nanometer scale by means of electron holography and Lorentz microscopy. In particular, the magnetization process in Alnico alloys was visualized for the first time by utilizing a sharp magnetic needle made of sintered $\mathrm{Nd}_{2} \mathrm{Fe}_{14} \mathrm{~B}$ in a transmission electron microscope. It was found that the direction of lines of magnetic flux changed at the boundaries between the $\alpha_{1}$ and $\alpha_{2}$ phases which were aligned in the direction of the magnetic field applied in the thermomagnetic treatment. In the Lorentz microscope image, these boundaries were observed as white lines and black bands, whose shapes reflected the difference in the shape anisotropy between Alnico 5 and Alnico 8. With an increase in the magnetic field induced by the magnetic needle, the magnetization direction of the domains magnetized in the direction opposite to that of the approaching needle was reversed, and finally a large reversed domain was formed. In both Alnico 5 and Alnico 8, it was shown that the magnetization process was accomplished through an entire magnetization reversal in each grain of the $\alpha_{1}$ phase.
\end{abstract}

(Received September 24, 2005; Accepted January 31, 2006; Published March 15, 2006)

Keywords: Alnico, electron holography, Lorentz microscopy, magnetization process

\section{Introduction}

Alnico alloys, which originated from the FeNiAl alloy invented by T. Mishima in 1931, were the first nanostructured permanent magnets. ${ }^{1,2)}$ Their magnetic properties were further improved by thermomagnetic treatment and addition of elements such as $\mathrm{Cu}$ and $\mathrm{Ti}$. Although more than 70 years have passed since Alnico alloys were developed, they continue to be widely used in applications such as relays, speed meters, motors, and loudspeakers, which require mechanical strength and temperature insensitivity. In particular, they are the only permanent magnets that can be used up to approximately $550^{\circ} \mathrm{C}$ because of their excellent thermal stability. ${ }^{2-6)}$

Alnico alloys are composed of two phases, a FeCo-rich phase $\left(\alpha_{1}\right)$ and a NiAl-rich phase $\left(\alpha_{2}\right)$, which are formed by spinodal decomposition. The size of the phases is approximately $20-40 \mathrm{~nm}$. Their magnetic properties depend on the magnetization difference between the two phases as well as the shape anisotropy in the ferromagnetic $\alpha_{1}$ phase. ${ }^{3-5)}$ It is well known that the magnetization mechanism in Alnico alloys is essentially based on a single domain process and that Alnico alloys have the configuration of interaction domains, which have been observed in magnets composed of nanometer scale small particles in the demagnetized state. ${ }^{2)}$ Thus, in order to improve magnetic properties such as the coercivity $\left(H_{\mathrm{c}}\right)$, remanence $\left(B_{\mathrm{r}}\right)$, and maximum energy product $\left[(B H)_{\max }\right]$, much research has focused not only on the control of the shape and distribution of the $\alpha_{1}$ and $\alpha_{2}$ phases, but also on the theoretical interpretation of the magnetization mechanism. ${ }^{7,8)}$ However, direct observation of the magnetization process at a nanometer scale has not yet been carried out, although it is indispensable for a complete understanding of the magnetic characteristics of nanostructured permanent magnets such as Alnico alloys. In this study,

*Corresponding author, E-mail: shindo@tagen.tohoku.ac.jp by utilizing a sharp magnetic needle made of sintered $\mathrm{Nd}_{2} \mathrm{Fe}_{14} \mathrm{~B}$ for inducing a strong magnetic field within a transmission electron microscope, ${ }^{9,10}$ the magnetization processes in Alnico 5 and Alnico 8 were visualized for the first time at a nanometer scale by in situ Lorentz microscopy combined with electron holography. Furthermore, the relationship between the magnetic domain structure and the microstructure is briefly discussed.

\section{Experimental Procedure}

Alnico 5 and Alnico 8, provided by NEOMAX Co., Ltd. were fabricated by the conventional casting method including thermomagnetic treatment. Their magnetic properties and compositions are listed in Table 1. Both electron holography and Lorentz microscopy experiments were carried out using a JEM-3000F transmission electron microscope with a special pole piece for shielding the magnetic field due to the objective lens. ${ }^{11)}$ Thin foil specimens for transmission electron microscopy observations were cut parallel to the direction of the magnetic field applied in the thermomagnetic treatment, and prepared using a focused ion-beam (JEM9310) to maintain uniform thickness of the specimens. In order to carry out in situ observations, the specimen and a sharp magnetic needle made of sintered $\mathrm{Nd}_{2} \mathrm{Fe}_{14} \mathrm{~B}$ were installed together in a special specimen holder controlled by a stepping motor and piezo drives, as shown in Fig. 1.9) With approaching the magnetic needle toward the specimen, the magnetization process was observed with a TV-system Gatan 676 using the Fresnel mode of Lorentz microscopy. ${ }^{10)}$ The coercivity $\left(H_{\mathrm{c}}\right)$, remanence $\left(B_{\mathrm{r}}\right)$, and maximum energy product $\left[(B H)_{\max }\right]$ of the magnetic needle used in this study were $1.1 \mathrm{MA} / \mathrm{m}, 1.39 \mathrm{~T}$, and $368 \mathrm{~kJ} / \mathrm{m}^{3}$, respectively. It is noted that the magnetic field at the top of the needle was estimated by simulation to be approximately $560 \mathrm{kA} / \mathrm{m}$, and its magnetization direction is shown in Fig. 1. ${ }^{10}$ 
Table 1 Magnetic properties and compositions of Alnico 5 and Alnico 8.

\begin{tabular}{|c|c|c|c|c|c|c|c|c|}
\hline \multirow{2}{*}{ No. } & \multirow{2}{*}{ Specimen } & \multirow{2}{*}{$\begin{array}{l}(B H)_{\max } \\
\left(\mathrm{kJ} / \mathrm{m}^{3}\right)\end{array}$} & \multirow{2}{*}{$\begin{array}{l}B_{\mathrm{r}} \\
(\mathrm{T})\end{array}$} & \multirow{2}{*}{$\begin{array}{c}H_{\mathrm{c}} \\
(\mathrm{kA} / \mathrm{m})\end{array}$} & \multicolumn{4}{|c|}{ Composition (mass\%) (balance Fe) } \\
\hline & & & & & $\mathrm{Al}$ & $\mathrm{Ni}$ & Co & $\mathrm{Cu}$ \\
\hline 1 & Alnico 5 & 42.3 & 1.30 & 50.5 & 8.0 & 14.1 & 24.0 & 3.0 \\
\hline 2 & Alnico 8 & 44.8 & 0.91 & 116 & 7.3 & 14.1 & 35.5 & 3.0 \\
\hline
\end{tabular}

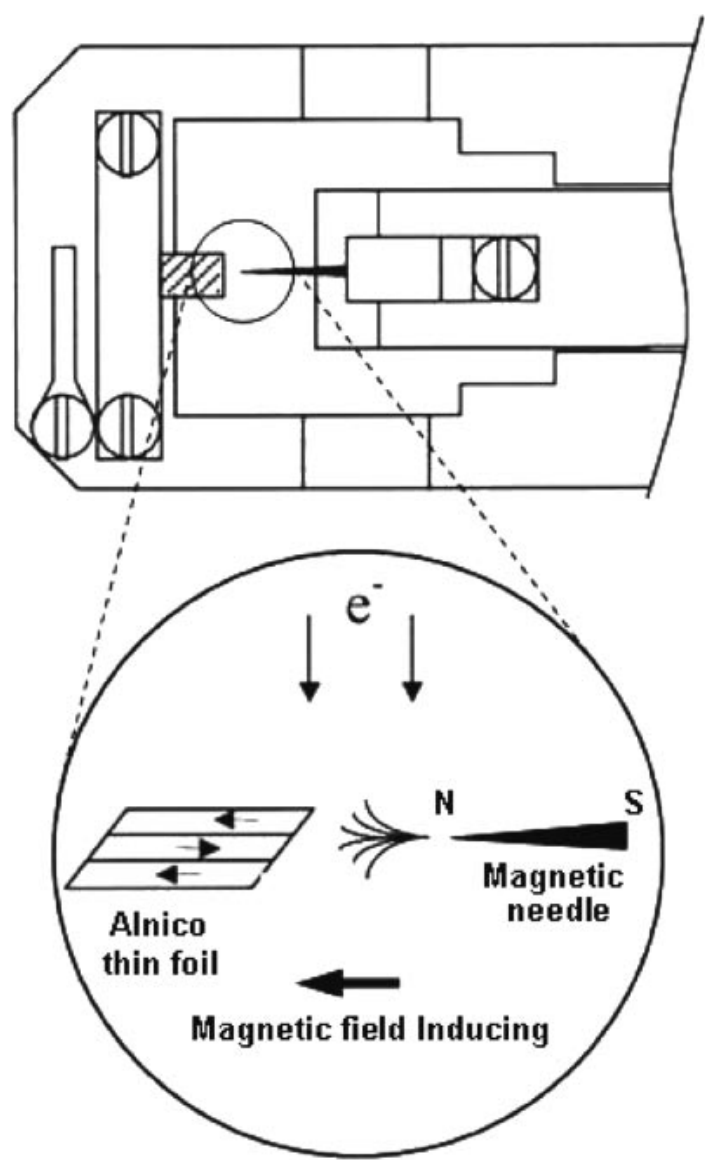

Fig. 1 Schematic illustration of a part of the specimen holder equipped with the sharp magnetic needle.

\section{Results and Discussion}

Figure 2(a) shows the dark-field image and electron diffraction pattern of demagnetized Alnico 8. In the brightfield image, it is not possible to distinguish the $\alpha_{1}$ phase from the $\alpha_{2}$ phase. Thus, the dark-field image was obtained using the 001 reflection in the corresponding electron diffraction pattern in Fig. 2(a) because the 001 reflection at [100] zone axis is only attributed to the $\alpha_{1}$ phase. ${ }^{12)}$ It is noted that the ferromagnetic $\alpha_{1}$ and nonmagnetic $\alpha_{2}$ phases appear to be bright and dark, respectively. As shown in the dark-field image, the $\alpha_{1}$ phases are well aligned in the direction of the magnetic field applied in the thermomagnetic treatment, as indicated by the white arrow, and have an elongated shape with a width of approximately $30 \mathrm{~nm}$. Figure 2(b) shows the Lorentz microscope image of demagnetized Alnico 8, which was observed using the Fresnel mode under a defocused condition. It can be seen that the white lines and black bands are aligned in the direction of the magnetic field applied in the thermomagnetic treatment. Generally, in the Fresnel mode, domain walls are observed as white lines or black bands because the electron beam passing through the domain is deflected by the Lorentz force, and thus the electron intensity increases or decreases at the domain wall according to the magnetization direction of the adjacent domains. ${ }^{13)}$ The Lorentz microscopy contrast formation can be explained in a similar way for the Alnico alloys with the ferromagnetic $\alpha_{1}$ and nonmagnetic $\alpha_{2}$ phases, and a schematic illustration is shown in Fig. 3. Figure 2(c) shows the phase distribution and reconstructed phase image that were obtained from the region indicated by the rectangle in Figs. 2(a) and (b). In the reconstructed phase image, the direction and density of white lines respectively correspond to the direction and density of lines of magnetic flux projected along the electron beam. We assumed that the contribution of electrostatic potential can be ignored since the specimens were prepared by a focused ionbeam technique. In Fig. 2(c), the direction of lines of magnetic flux tends to change at the boundaries between the $\alpha_{1}$ and the $\alpha_{2}$ phases as a result of the stray field of the ferromagnetic $\alpha_{1}$ phase that is thought to be a single domain. In addition, we should note that the distribution of lines of magnetic flux in Fig. 2(c) shows the detailed magnetic domain structure, and it includes the information from the Lorentz microscope image in Fig. 2(b). Thus, taking into account both the dark-field image and the reconstructed phase image, it is clear that the boundaries indicated by the gray arrowheads in Fig. 2(c) correspond to white lines and a black band indicated by the gray arrowheads in Fig. 2(b). Therefore, we can consider the white lines and black bands observed by Lorentz microscopy are the boundaries where the direction of lines of magnetic flux changes at all the boundaries between the $\alpha_{1}$ and the $\alpha_{2}$ phases (hereinafter referred to as the interaction domain boundary $\left.{ }^{2,3)}\right)$. The Lorentz microscope image of demagnetized Alnico 5 is shown in Fig. 2(d). The white lines and black bands, indicating the interaction domain boundaries, have nearly the same direction as the direction of the magnetic field applied in the thermomagnetic treatment, as shown in Fig. 2(b). However, the interaction domain boundaries observed in Alnico 8 are almost straight, as shown in Fig. 2(b), while they appear as fluctuated line-shapes in Alnico 5, as shown in Fig. 2(d). It is noted that Alnico 8 has a higher coercivity than Alnico 5, as shown in Table 1, which is attributed to the large shape anisotropy in the $\alpha_{1}$ phase. Therefore, it is reasonably considered that the magnetic bamboo-like microstructure in Alnico 8 reflects the fact that Alnico 8 has larger shape anisotropy than Alnico 5.

Figure 4 shows Lorentz microscope images, captured from videotape, that show the magnetization process in Alnico 5. The distance between the magnetic needle and the specimen is indicated in the bottom right corner. In the initial stage [Fig. 4(a)], it can be seen that the interaction domain 

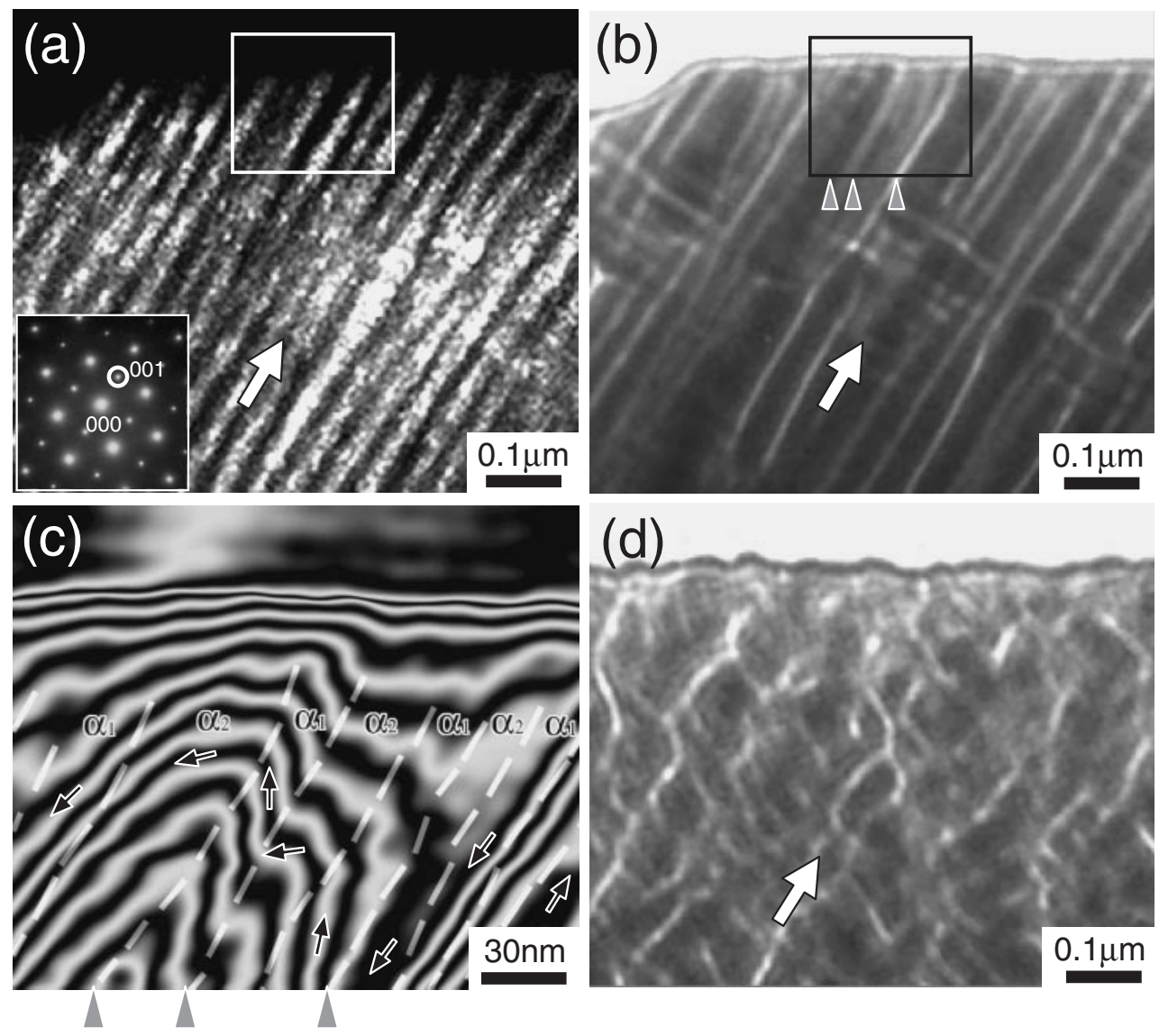

Fig. 2 (a) Dark-field image and (b) Lorentz microscope image of demagnetized Alnico 8. (c) Reconstructed phase image obtained from the region indicated by the rectangle in Figs. 2(a) and (b). Broken white lines indicate the boundaries between the $\alpha_{1}$ and $\alpha_{2}$ phases. (d) Lorentz microscope image of demagnetized Alnico 5. The white arrow indicates the direction of the magnetic field applied in the thermomagnetic treatment, and black arrows indicate the direction of lines of magnetic flux.

boundaries have a complex structure similar to that of the demagnetized state shown in Fig. 2(d). With an increase in the magnetic field induced by the magnetic needle, the area of domains magnetized in the direction opposite to that of the approaching needle decreases while that of domains magnetized in the same direction increases [Figs. 4(b) and (c)]. Finally, the long rod-type domains disappear suddenly and the magnetization reversal is completed in Fig. 4(d), where the magnetic field induced by the magnetic needle was estimated by simulation to be approximately $80 \mathrm{kA} / \mathrm{m}$. Interestingly, a white contrast with a width of approximately $40 \mathrm{~nm}$ is observed in Fig. 4(d) although a reversed domain is formed. This contrast is thought to result from the deflection of the electron beam passing through the ferromagnetic $\alpha_{1}$ phase.

Figures 5(a) and (b) show enlarged Lorentz microscope images of Alnico 5, captured from videotape. The time difference between Figs. 5(a) and (b) is 0.066 seconds, and the distance between the specimen and magnetic needle, where the induced magnetic field was estimated by simulation to be approximately $56 \mathrm{kA} / \mathrm{m}$, is about $50 \mu \mathrm{m}$. Figure 5(c) is a schematic illustration showing the magnetization process caused by the magnetic field induced by the magnetic needle. The interaction domain boundaries are indicated by the black and white lines. In Fig. 5(c), it can be observed that the magnetization direction of the region indicated by "R" was reversed; therefore, the boundary situated at the position indicated by broken black lines moves toward a new position shown by the solid black line. On the other hand, the distance between the solid black line and white line was evaluated to be approximately $40 \mathrm{~nm}$, which is equal not only to the size of the domain seen as a long rodtype domain in Fig. 4(c) but also the size of the ferromagnetic $\alpha_{1}$ phase reported many times in the literatures. ${ }^{3-5}$ ) Consequently, the magnetization process is accomplished through the magnetization reversal of the $\alpha_{1}$ phase. By taking into account the distance between the solid black and white line, the mechanism of magnetization reversal can be regarded as a reversal of the entire magnetization in one grain of the $\alpha_{1}$ phase through, presumably, the incoherent rotation mode. ${ }^{3,4)}$

Figures 6(a) and (b) show the reconstructed phase images of Alnico 8 before and after a magnetic field of approximately $110 \mathrm{kA} / \mathrm{m}$ was induced by the magnetic needle. The reconstructed phase images were obtained when the magnetic needle had been removed. Figures 6(c) and (d) show schematic illustrations of Figs. 6(a) and (b), respectively. After the magnetic field was induced, a change in the direction of lines of magnetic flux was observed at the regions indicated by " $R$ ". This result is attributed to the magnetization reversal of domains caused by the magnetic field induced by the magnetic needle, which is similar to the 
(a)

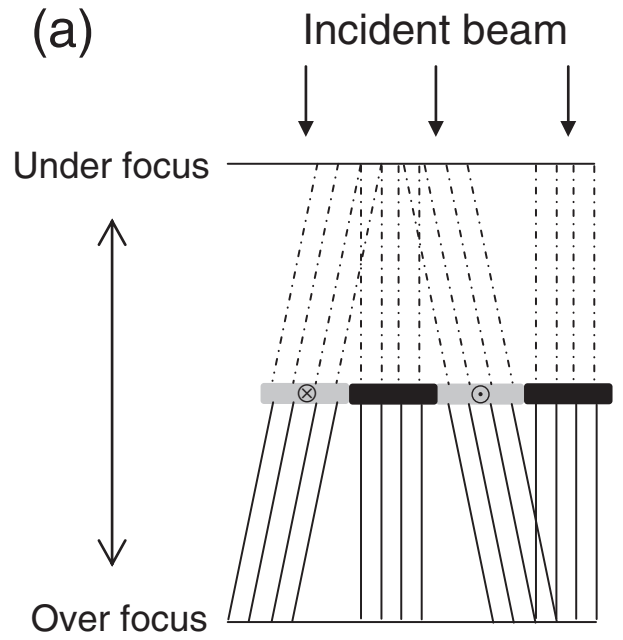

(b)

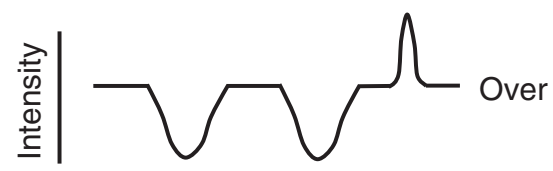

(c)

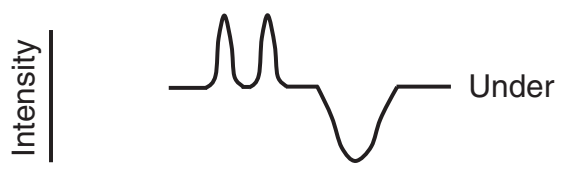

Fig. 3 (a) Schematic illustration showing contrast formation in Lorentz microscopy. The grey and black regions indicate the ferromagnetic $\alpha_{1}$ and nonmagnetic $\alpha_{2}$ phases, respectively. Intensity distributions are shown for the overfocused (b) and the underfocused condition (c).
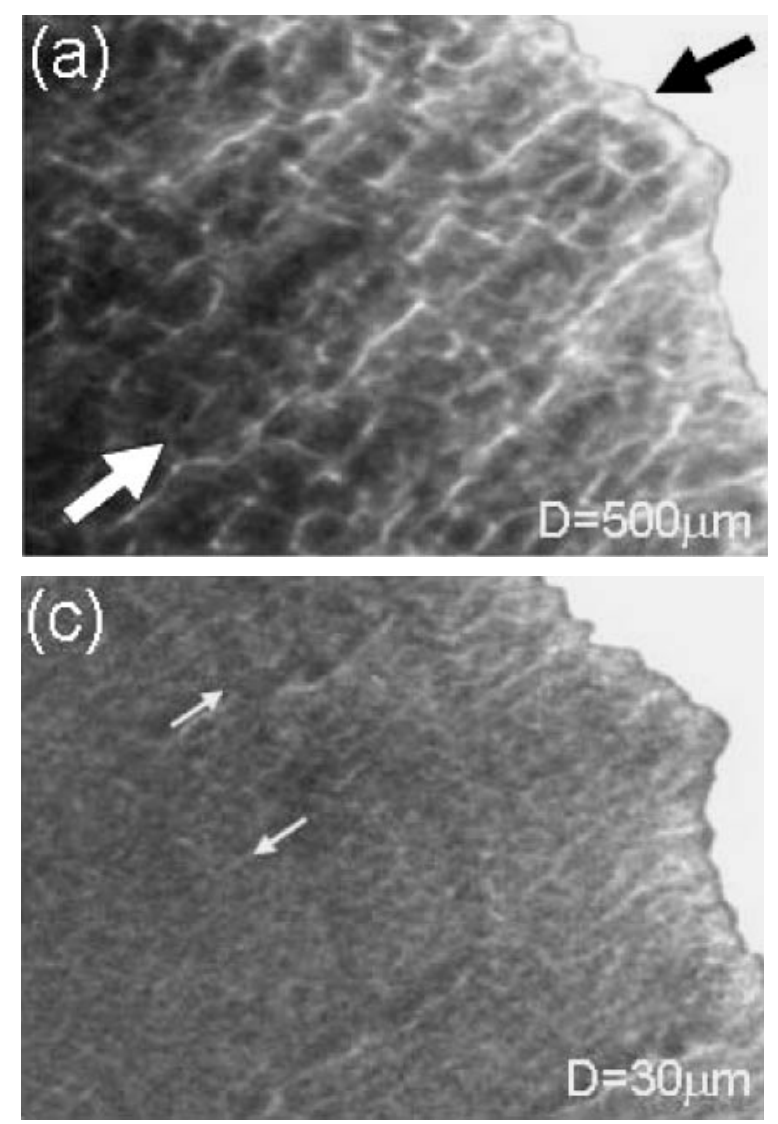

magnetization process in Alnico 5. In Figs. 6(b) and (d), it is noted that the width of the domains, where the direction of lines of magnetic flux is opposite to the direction of the approaching needle (indicated by the small white arrows) is evaluated to be approximately $30 \mathrm{~nm}$, corresponding to the width of the $\alpha_{1}$ phase shown in Fig. 2(a). Consequently, it is considered that the magnetization reversal in each elongated grain of the $\alpha_{1}$ phase is accomplished in the incoherent rotation mode, as in the case of Alnico 5.

\section{Conclusions}

The in situ observations of the magnetization process in Alnico 5 and Alnico 8 by electron holography and Lorentz microscopy are summarized as follows:

(1) The $\alpha_{1}$ phases were aligned in the direction of the magnetic field applied in the thermomagnetic treatment. It was concluded that white lines and black bands observed by Lorentz microscopy corresponded to the boundaries, referred to in this study as the interaction domain boundaries, where the direction of lines of magnetic flux changed at the boundaries between the $\alpha_{1}$ and the $\alpha_{2}$ phases.

(2) In the demagnetized state, it was found that the interaction domain boundaries appeared as fluctuated line-shapes in Alnico 5 while those observed in Alnico 8 were almost straight, reflecting the difference in the shape anisotropy, that is coercivity.
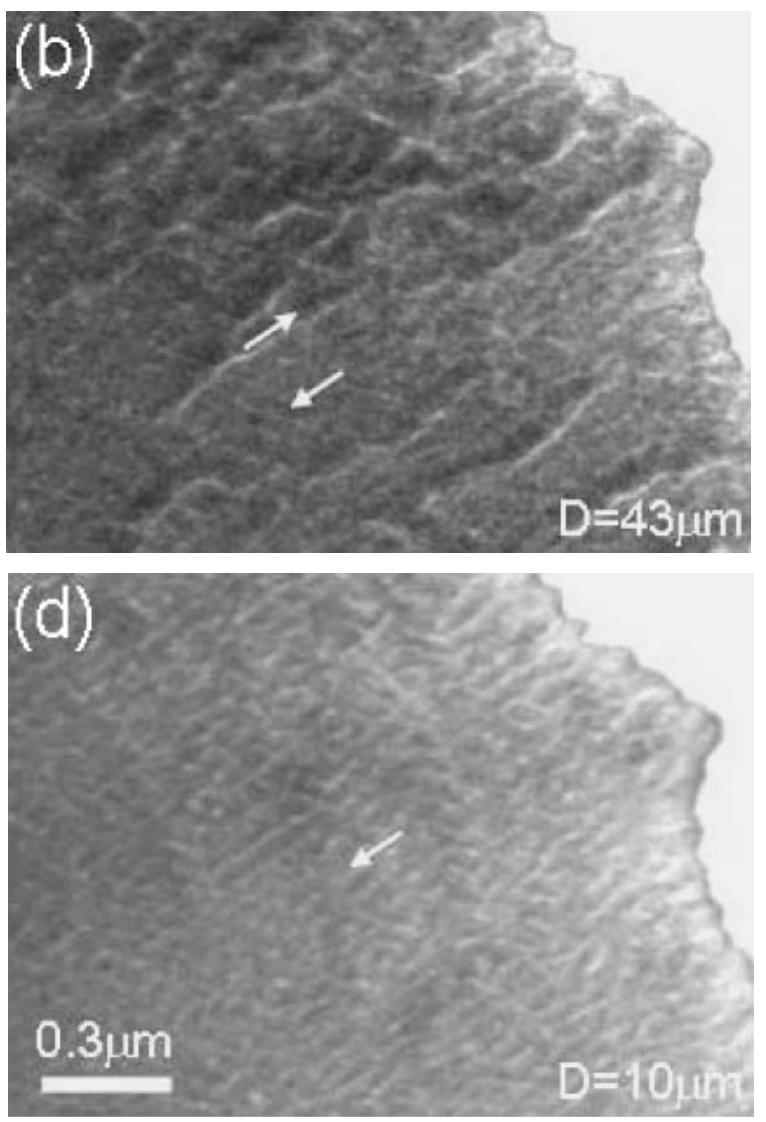

Fig. 4 Lorentz microscope images showing the magnetization process in Alnico 5 captured from videotape. Large black and white arrows indicate the direction of approach of the magnetic needle and the direction of the magnetic field applied in the thermomagnetic treatment, respectively. Small white arrows indicate the magnetization direction. 

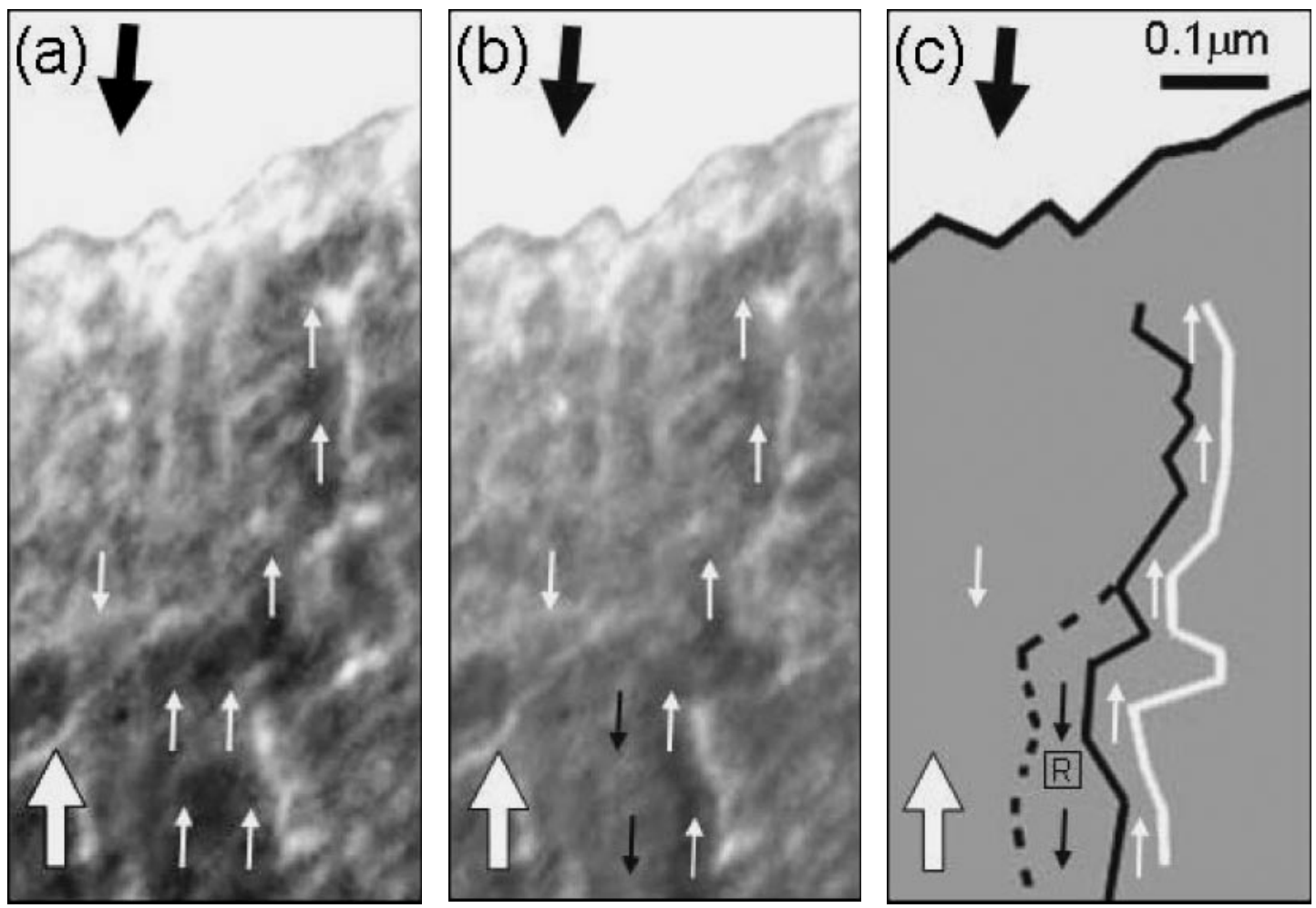

Fig. 5 (a) and (b) Enlarged Lorentz microscope images of Alnico 5 captured from videotape. The distance between the specimen and magnetic needle is $50 \mu \mathrm{m}$, and the time difference between (a) and (b) is $0.066 \mathrm{~s}$. (c) Schematic illustration showing the magnetization process caused by the magnetic field induced by the magnetic needle. Large black and white arrows indicate the direction of approach of the magnetic needle and the direction of the magnetic field applied in the thermomagnetic treatment, respectively. Small black and white arrows indicate the magnetization direction; in particular, the magnetization direction at the magnetization reversed domain is indicated by small black arrows.
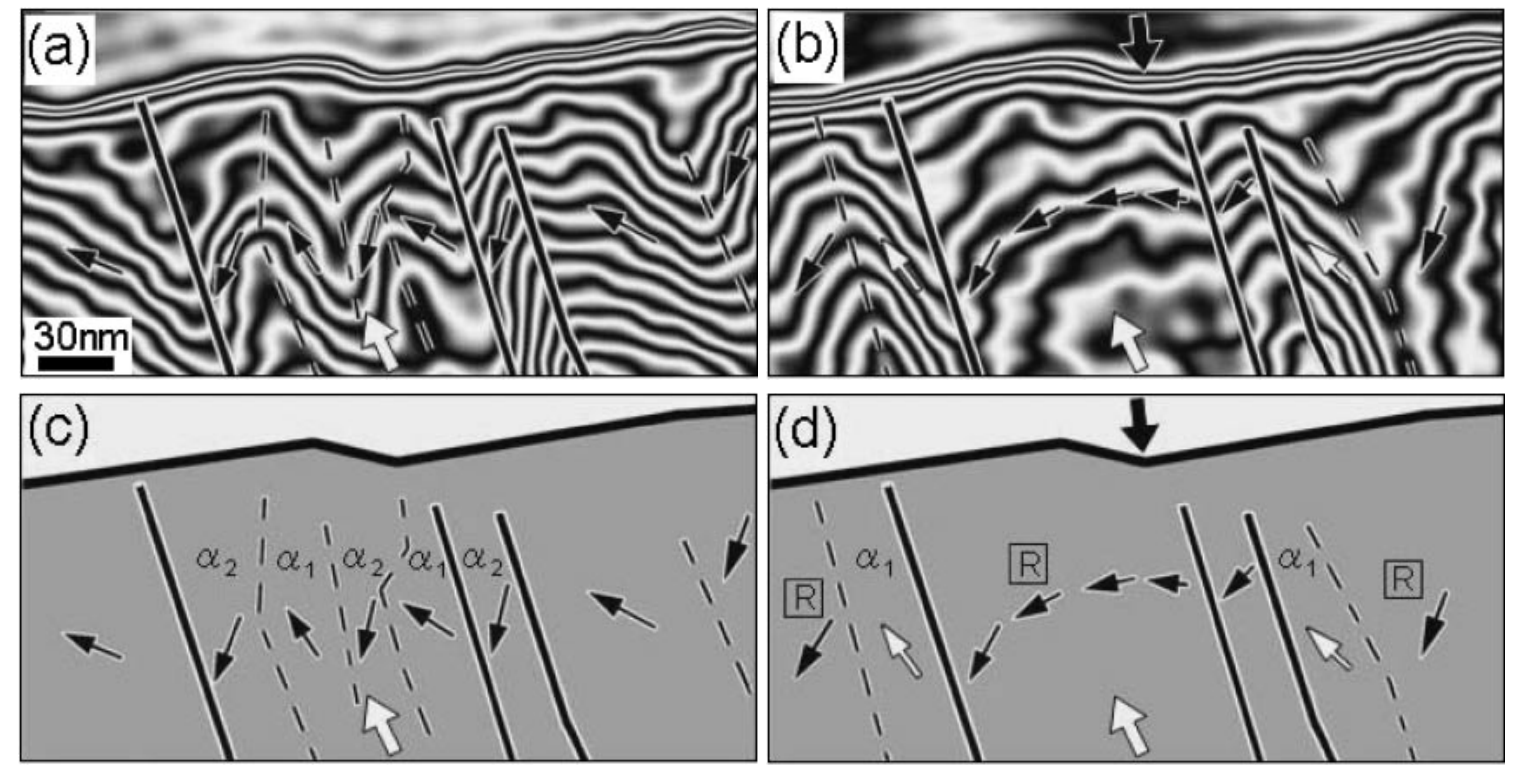

Fig. 6 (a) Reconstructed phase image of demagnetized Alnico 8. (b) Reconstructed phase image after inducing a magnetic field using the magnetic needle. (c) and (d) are schematic illustrations of (a) and (b), respectively. Solid and broken black lines indicate the interaction domain boundaries. The interaction domain boundaries that are not changed by inducing a magnetic field are depicted by solid black lines. Small black and white arrows indicate the direction of lines of magnetic flux. Large black and white arrows indicate the direction of approach of the magnetic needle and the direction of the magnetic field applied in the thermomagnetic treatment, respectively.

(3) The magnetization process in Alnico alloys was visualized. With an increase in the magnetic field induced by the magnetic needle, the magnetization direction of domains magnetized in the direction opposite to that of the approaching needle was reversed, and finally a large reversed domain was formed. 
(4) In both Alnico 5 and Alnico 8, it was shown that the magnetization process was accomplished through an entire magnetization reversal in each grain of the $\alpha_{1}$ phase.

\section{Acknowledgments}

This work was partly supported by the financial support from a Grant-in-Aid for Scientific Research (A) from Japan Society for the Promotion of Science.

\section{REFERENCES}

1) T. Mishima: British patent, 378478-1931.

2) A. Hubert and R. Schäfer: Magnetic Domains (Springer, 1998) 550 560 .

3) B. D. Cullity: Introduction to Magnetic Materials (Addison-Wesley,
1972) 565-575.

4) E. P. Wohlfarth: Ferromagnetic Materials, Vol. 3 (North-Holland Publishing Company, 1982) 107-188.

5) R. C. O'Handley: Modern Magnetic Materials (John Wiley \& Sons, 1999) 476-484.

6) A. Goldman: Handbook of Modern Ferromagnetic Materials (Kluwer Academic Publisher, 1999) 75-86.

7) D. J. Craik and R. Lane: J. Phys. D 2 (1968) 33-45.

8) P. Campbell: IEEE Trans. Magn. MAG-18 (1982) 898-904.

9) D. Shindo, Y.-G. Park, Y. Gao and H. S. Park: J. Appl. Phys. 95 (2004) 6521-6526.

10) H. S. Park, Y.-G. Park, Y. Gao, D. Shindo and M. Inoue: J. Appl. Phys. 97 (2005) 033908-1-033908-4.

11) D. Shindo, Y.-G. Park, Y. Murakami, Y. Gao, H. Kanekiyo and S. Hirosawa: Scr. Mater. 48 (2003) 851-856.

12) A. Hütten, G. Reiss, W. Saikaly and G. Thomas: Acta Mater. 49 (2001) 827-835.

13) D. Shindo and T. Oikawa: Analytical Electron Microscopy for Materials Science (Springer, 2002) 111-113. 